\title{
The Chlorine Nuclear Quadrupole Coupling Tensor in Chlorotrifluoroethylene
}

\author{
K. W. Hillig II, E. R. BitTNeR, AND R. L. KUCZKOWSKI
}

Department of Chemistry, University of Michigan, Ann Arbor, Michigan 48109

W. LEWIS-BEVAN

Department of Chemistry and Biochemistry, Southern Illinois University, Carbondale, Illinois 62901

\author{
AND \\ M. C. L. GerRY \\ Department of Chemistry. The University of British Columbia, 2036 Main Mall, \\ Vancouver, British Columbia, Canada, V6T 1 Y6
}

\begin{abstract}
The 273 hyperfine components from 56 rotational transitions of chlorotrifluoroethylene were measured with a Fourier transform microwave spectrometer. A global least-squares fit was made to the rotational constants, quartic distortion constants, and ${ }^{35} \mathrm{Cl}$ quadrupole coupling constants; the RMS deviation of the fit was $1.6 \mathrm{kHz}$. It is shown that such high-resolution measurements enable $x_{a b}$ to be determined without the near degeneracies usually necessary with less precise data. The principle tensor quadrupole coupling constants are $x_{z z}=-77.46(10), x_{x x}=38.85(10)$, and $x_{y y}=x_{c c}=38.614$ (3) $\mathrm{MHz}$. The values are comparable to other vinyl chlorides, removing some anomalies from previous studies. (c) 1988 Academic Press, Inc.
\end{abstract}

\section{INTRODUCTION}

The microwave spectrum of chlorotrifluoroethylene, $\mathrm{CF}_{2}=\mathrm{CFCl}$, was first reported by Stone and Flygare (1). They measured several low- $J Q$ - and $R$-branch transitions with a Stark-modulated spectrometer and used them to evaluate the rotational constants of the ${ }^{35} \mathrm{Cl}$ isotopic species, along with a structure consistent with the limited isotopic data. The ${ }^{35} \mathrm{Cl}$ quadrupole coupling constants in the inertial axis system $\left(x_{a a}, x_{b b}\right.$, and $x_{c c}$ ) were also determined. Using this structure and the assumption that the $\mathrm{C}-\mathrm{Cl}$ bond is a principal axis of the quadrupole tensor, the inertial quadrupole tensor was rotated to obtain the principal value $x_{z z}=-89.1 \mathrm{MHz}$. This value was much larger than those of other vinyl halides, and the result was rationalized in terms of the bonding of the molecule. A second MW study of this compound appeared subsequently and reported a quite different assignment and rotational constants and more normal quadrupole coupling (2).

We were motivated initially to explore the assignment ambiguity, and also to resolve the question of the anomalous quadrupole coupling. The latter could be achieved if the complete quadrupole tensor could be determined, in particular the off-diagonal 
element $x_{a b}$. This constant would permit the quadrupole tensor to be diagonalized directly without any structural assumptions. To determine $x_{a b}$, however, deviations from first-order quadrupole splitting patterns must be measured. For ${ }^{35} \mathrm{Cl}$ these deviations are usually so small that in the absence of accidental near degeneracies of the correct type (see, for example Ref. (4)) they cannot be determined with a Starkmodulated waveguide spectrometer.

\section{EXPERIMENTAL DETAILS}

The transitions were observed with the FTMW spectrometer at the University of Michigan. This is of the Balle-Flygare type and operates in the frequency range of 6$18 \mathrm{GHz}(3,5)$. Both a modified Bosch 0-280-150-045 fuel injector and a Newport Research Model BV-100 pulsed gas valve were used as molecular beam sources, with orifice diameters of 0.5 and $1.0 \mathrm{~mm}$. A sample of $1 \% \mathrm{CF}_{2}=\mathrm{CFCl}$ in argon was used at a backing pressure of 1-2 atm.

Most transitions could be seen by averaging $1-100$ pulses, although $10^{3}-10^{5}$ pulses were typically accumulated when determining final transition frequencies. Doppler splitting of the lines was minimized by careful adjustment of the timing of gas and MW pulses. Typical linewidths were $\approx 10 \mathrm{kHz}$ (FWHM) and frequency measurements were usually reproducible to within $1 \mathrm{kHz}$. Transitions were detectable for states with rotational energies as high as $6.8 \mathrm{~cm}^{-1}(9.8 \mathrm{~K})$. Such energies imply less efficient rotational cooling than with other species which have been studied with this instrument; no special efforts were made to generate states this high in energy or to detect them other than with long signal averaging times for the weakest transitions.

The program used to fit the transitions performs a simultaneous least-squares fit to the rotational, centrifugal distortion and quadrupole coupling constants including $x_{a b}$. It has been previously applied to species such as BrNCO (6) and chloroketene (4).

\section{RESULTS AND ANALYSIS}

Initially a search was made for four low- $J(J \leqslant 3)$ lines of the ${ }^{35} \mathrm{Cl}$ species. It quickly became clear that the constants of Ref. ( $I$ ) predicted the transition frequencies and splitting patterns fairly well while those of Ref. (2) did not. However, the splittings deviated from a simple rigid rotor plus quadrupole Hamiltonian with diagonal coupling constants by several tens of kiloHertz. Including $x_{a b}$ in the fit did not reproduce the frequencies to within experimental error and gave an unreasonable value of $x_{a b}$. A second fit was tried, including the measurements of Stone and Flygare in the data set, and the cause of the earlier discrepancy became clear. The accuracy of the FTMW data is so high that unless the positions of the rotational energy levels are very well determined, including centrifugal distortion contributions, anomalous fits are obtained even at very low $J$. Thus, in order to determine accurate quadrupole constants, accurate distortion constants were needed. These could be obtained only by drastically increasing the number of measured transitions.

In the end, 273 components of 56 rotational transitions of $\mathrm{CF}_{2}=\mathrm{CF}^{35} \mathrm{Cl}$ were measured with the FTMW spectrometer. These have included remeasurements of those reported earlier (1). A global least-squares fit was made to all rotational constants, quartic distortion constants (Watson's S-reduction in the $I^{r}$ representation (7)), and 
${ }^{35} \mathrm{Cl}$ quadrupole coupling constants. The results are presented in Table I. Several fits were attempted including in addition various sextic distortion constants; in all cases these were indeterminate and the goodness of the fit was unaffected. The very small observed linewidths and RMS deviations suggest that effects of magnetic spin-rotation coupling were also negligible.

It is clear from Table I that the precision of the derived constants is extremely high. The precision in $x_{a b}$ in particular is higher than has ever been previously reported (4). Table II presents the frequencies of all measured transitions along with a comparison of the obs - calc deviations obtained when $x_{a b}$ is included in the fitting process and when it is deleted. The differences represent the contributions from $x_{a b}$.

Although the contributions of $x_{a b}$ to the frequencies are small in general $(<0.1$

TABLE I

Spectroscopic Constants of $\mathrm{CF}_{2}=\mathrm{CF}^{35} \mathrm{Cl}$

\begin{tabular}{|c|c|c|c|}
\hline & \multirow[b]{2}{*}{ Ref. (1 $)$} & \multicolumn{2}{|c|}{ Present Work } \\
\hline & & Final values & Reduced Data Set ${ }^{a}$ \\
\hline \multicolumn{4}{|c|}{ Rotational constants (MHz) } \\
\hline A & $4506.05(2)^{\mathrm{b}}$ & $4506.105428(88)^{C}$ & $4506.10546(10)^{c}$ \\
\hline B & $2268.66(2)$ & $2268.700337(50)$ & $2268.700412(86)$ \\
\hline $\mathrm{c}$ & $1508.09(2)$ & $1508.093176(51)$ & $1508.09323 .3(87)$ \\
\hline \multicolumn{4}{|c|}{ Centrifugal distortion constants (kHz) } \\
\hline $\mathrm{D}_{\mathrm{J}}$ & d & $0.21266(89)$ & $0.2153(28)$ \\
\hline $\mathrm{D}_{\mathrm{JK}}$ & d & $0.0626(32)$ & $0.0637(38)$ \\
\hline $\mathrm{D}_{\mathrm{K}}$ & d & $1.1152(72)$ & $1.1094(85)$ \\
\hline$d_{1}$ & d & $-0.09325(72)$ & $-0.09338(89)$ \\
\hline$d_{2}$ & d & $-0.01740(33)$ & $-0.01734(41)$ \\
\hline \multicolumn{4}{|c|}{${ }^{35} \mathrm{Cl}$ quadrupole coupling constants (MHz) } \\
\hline$x_{\mathrm{aa}}$ & $-50.10(10)$ & $-49.84817(83)$ & $-49.84815(86)$ \\
\hline$x_{b b}-x_{c c}$ & $-27.38(22)$ & $-27.3799(17)$ & $-27.3799(18)$ \\
\hline$\left|x_{a b}\right| \cdot$ & d & $49.489(34)$ & $49.48(11)$ \\
\hline \multicolumn{4}{|c|}{ r.m.s. deviation (MHz) } \\
\hline & d & 0.0016 & 0.0016 \\
\hline \multicolumn{4}{|c|}{$\begin{array}{l}\text { Least squares fit did not include the four transitions with the biggest } \\
\text { contributions to } \chi_{\mathrm{ab}} \text { (see text). } \\
b_{\text {For ref. (1), numbers in parentheses are the stated uncertainties in units }} \\
\text { of the last significant figures. } \\
c_{\text {For the present work, numbers in parentheses are one standard deviation in }} \\
\text { units of the last significant figures. } \\
d_{\text {Not determined or stated }}\end{array}$} \\
\hline
\end{tabular}


TABLE II

Measured Rotational Transitions (in $\mathrm{MHz}$ ) of $\mathrm{CF}_{2} \mathrm{CFCl}$

\begin{tabular}{|c|c|c|c|c|c|c|}
\hline \multicolumn{2}{|c|}{$\begin{array}{l}\text { Transition } \\
F^{\prime}-F^{n}\end{array}$} & \multicolumn{2}{|r|}{$\begin{array}{l}\text { Normal ised } \\
\text { Weight }\end{array}$} & \multirow[t]{2}{*}{$\begin{array}{l}\text { Observed } \\
\text { Frequency }\end{array}$} & \multirow[t]{2}{*}{ without ${ }^{\text {Res }}{ }_{\mathrm{ab}}$} & $\begin{array}{l}\text { Residuals }{ }^{2} \\
{ }_{\mathrm{ab}} \text { with } x_{\mathrm{ab}}\end{array}$ \\
\hline a-type & transit & ior & & & & \\
\hline $\begin{array}{l}202^{2} \\
3 / 2 \\
7 / 2 \\
5 / 2 \\
1 / 2 \\
3 / 2 \\
5 / 2 \\
3 / 2\end{array}$ & $\begin{array}{l} \\
-\quad 3 / 2 \\
-\quad 3 / 2 \\
-\quad 5 / 2 \\
-\quad 3 / 2 \\
-\quad 1 / 2 \\
-\quad 5 / 2 \\
-\quad 5 / 2 \\
-\quad 1 / 2\end{array}$ & 1 & $\begin{array}{r}1.000 \\
1.000 \\
1.000 \\
1.000 \\
1.000 \\
1.000 \\
1.000 \\
1.000\end{array}$ & $\begin{array}{l}7413.466 \\
7400.434 \\
7391.615 \\
7391.076 \\
7391.054 \\
7387.939 \\
7378.581 \\
7378.023\end{array}$ & $\begin{array}{l}0.071 \\
0.077 \\
0.005 \\
0.055 \\
0.094 \\
0.040 \\
0.017 \\
0.101\end{array}$ & $\begin{array}{l}0.0 \\
-0.001 \\
0.001 \\
0.001 \\
0.0 \\
-0.002 \\
-0.001 \\
0.0\end{array}$ \\
\hline $\begin{array}{c}21 / 2{ }^{2} \\
1 / 2 \\
7 / 2 \\
3 / 2 \\
3 / 2 \\
3 / 2 \\
5 / 2 \\
5 / 2\end{array}$ & $\begin{array}{l}- \\
1 / 2 \\
3 / 2 \\
5 / 2 \\
1 / 2 \\
5 / 2 \\
3 / 2 \\
5 / 2 \\
3 / 2\end{array}$ & 1 & $\begin{array}{r}1.000 \\
1.000 \\
1.000 \\
1.000 \\
1.000 \\
1.000 \\
1.000 \\
1.000\end{array}$ & $\begin{array}{l}6805.402 \\
6800.374 \\
6796.303 \\
6795.756 \\
6793.575 \\
6790.726 \\
6786.659 \\
6783.811\end{array}$ & $\begin{array}{r}-0.067 \\
-0.038 \\
0.002 \\
-0.049 \\
0.021 \\
-0.022 \\
0.002 \\
-0.041\end{array}$ & $\begin{array}{r}-0.001 \\
0.0 \\
0.002 \\
0.001 \\
-0.003 \\
-0.001 \\
-0.002 \\
0.001\end{array}$ \\
\hline $\begin{array}{l}21 / 2 \\
7 / 2 \\
5 / 2 \\
3 / 2 \\
5 / 2 \\
3 / 2 \\
1 / 2\end{array}$ & $\begin{array}{ll} & - \\
- & 5 / 2 \\
- & 3 / 2 \\
- & 1 / 2 \\
- & 5 / 2 \\
- & 3 / 2 \\
- & 1 / 2\end{array}$ & 1 & $\begin{array}{r}1.000 \\
1.000 \\
1.000 \\
1.000 \\
1.000 \\
1.000\end{array}$ & $\begin{array}{l}8316.929 \\
8304.491 \\
8323.888 \\
8314.130 \\
8306.498 \\
8326.700\end{array}$ & $\begin{array}{l}0.007 \\
0.019 \\
0.028 \\
0.005 \\
0.014 \\
0.023\end{array}$ & $\begin{array}{r}0.001 \\
0.002 \\
0.006 \\
-0.001 \\
-0.001 \\
0.0\end{array}$ \\
\hline $\begin{array}{l}30^{3}{ }^{3} \\
5 / 2 \\
9 / 2 \\
7 / 2 \\
3 / 2 \\
5 / 2 \\
7 / 2\end{array}$ & $\begin{array}{ll} & - \\
- & 3 / 2 \\
- & 5 / 2 \\
- & 7 / 2 \\
- & 5 / 2 \\
- & 1 / 2 \\
- & 3 / 2 \\
- & 7 / 2\end{array}$ & 2 & $\begin{array}{r}02 \\
1.000 \\
1.000 \\
1.000 \\
1.000 \\
1.000 \\
1.000 \\
1.000\end{array}$ & $\begin{array}{l}10732.775 \\
10728.283 \\
10722.594 \\
10721.828 \\
10719.743 \\
10718.927 \\
10708.792\end{array}$ & $\begin{array}{r}-0.007 \\
0.003 \\
0.004 \\
0.004 \\
-0.001 \\
-0.017 \\
0.015\end{array}$ & $\begin{array}{l}0.0 \\
-0.003 \\
0.002 \\
0.002 \\
-0.001 \\
0.0 \\
-0.001\end{array}$ \\
\hline $\begin{array}{l}3{ }^{1}{ }^{3} \\
5 / 2 \\
9 / 2 \\
3 / 2 \\
7 / 2 \\
5 / 2 \\
7 / 2\end{array}$ & $\begin{array}{ll} & - \\
- & 3 / 2 \\
- & 5 / 2 \\
- & 7 / 2 \\
- & 1 / 2 \\
- & 5 / 2 \\
- & 3 / 2 \\
- & 7 / 2\end{array}$ & 2 & $\begin{array}{r}121.000 \\
1.000 \\
1.000 \\
1.000 \\
1.000 \\
1.000 \\
1.000\end{array}$ & $\begin{array}{l}10106.814 \\
10100.754 \\
10097.972 \\
10097.167 \\
10094.719 \\
10093.838 \\
10085.078\end{array}$ & $\begin{array}{l}0.050 \\
0.032 \\
0.003 \\
0.067 \\
0.035 \\
0.013 \\
0.038\end{array}$ & $\begin{array}{l}0.0 \\
0.0 \\
0.002 \\
0.0 \\
0.002 \\
0.001 \\
0.001\end{array}$ \\
\hline $\begin{array}{l}3{ }^{1} / 2 \\
3 / 2 \\
9 / 2\end{array}$ & $\begin{array}{ll} & - \\
- & 3 / 2 \\
- & 1 / 2 \\
- & 7 / 2\end{array}$ & 2 & $\begin{array}{r}1.000 \\
1.000 \\
1.000\end{array}$ & $\begin{array}{l}12360.652 \\
12357.834 \\
12356.960\end{array}$ & $\begin{array}{l}0.032 \\
0.031 \\
0.004\end{array}$ & $\begin{array}{l}0.001 \\
0.0 \\
0.0\end{array}$ \\
\hline
\end{tabular}

1 Measurements were weighted accoraing to $1 / \sigma^{2}$, where $\sigma$ is the uncertainty in the measurements. Unit weight corresponded to an uncertainty of $0.001 \mathrm{MHz}$.

2 Observed Erequency minus the frequency calculated using the constants in Table I. 
TABLE II-Continued

\begin{tabular}{|c|c|c|c|c|c|c|c|}
\hline \multirow{2}{*}{\multicolumn{2}{|c|}{$\begin{array}{l}\text { Trans } \\
F^{\prime}\end{array}$}} & & \multirow{2}{*}{\multicolumn{2}{|c|}{$\begin{array}{c}\text { Normal ised } \\
\text { Weight }\end{array}$}} & \multirow{2}{*}{$\begin{array}{l}\text { Observed } \\
\text { Frequency }\end{array}$} & \multicolumn{2}{|c|}{ Residuals } \\
\hline & & & & & & a & $n x_{a b}$ \\
\hline $\begin{array}{l}5 / 2 \\
5 / 2 \\
7 / 2 \\
7 / 2\end{array}$ & $\begin{array}{l}- \\
- \\
-\end{array}$ & $\begin{array}{l}5 / 2 \\
3 / 2 \\
5 / 2 \\
7 / 2\end{array}$ & & $\begin{array}{l}1.000 \\
1.000 \\
1.000 \\
1.000\end{array}$ & $\begin{array}{l}12356.497 \\
12354.488 \\
12353.641 \\
12350.842\end{array}$ & $\begin{array}{l}0.007 \\
0.009 \\
0.019 \\
0.017\end{array}$ & $\begin{array}{c}-0.001 \\
0.0 \\
0.0 \\
-0.002\end{array}$ \\
\hline $\begin{array}{l}39^{2}{ }^{2} \\
7 / 2 \\
5 / 2 \\
3 / 2\end{array}$ & $\begin{array}{l}- \\
- \\
-\end{array}$ & $\begin{array}{l}- \\
7 / 2 \\
5 / 2 \\
3 / 2 \\
1 / 2\end{array}$ & & $\begin{array}{r}21.000 \\
1.000 \\
1.000 \\
1.000\end{array}$ & $\begin{array}{l}11333.954 \\
11321.476 \\
11330.431 \\
11342.810\end{array}$ & $\begin{array}{r}0.020 \\
0.003 \\
0.044 \\
-0.023\end{array}$ & $\begin{array}{l}0.001 \\
0.001 \\
0.001 \\
0.002\end{array}$ \\
\hline $\begin{array}{l}32^{2} 1 \\
3 / 2 \\
7 / 2 \\
9 / 2 \\
5 / 2 \\
3 / 2 \\
7 / 2 \\
5 / 2\end{array}$ & $\begin{array}{l}- \\
- \\
- \\
- \\
- \\
-\end{array}$ & $\begin{array}{l}- \\
1 / 2 \\
7 / 2 \\
7 / 2 \\
3 / 2 \\
3 / 2 \\
5 / 2 \\
5 / 2\end{array}$ & 2 & $\begin{array}{r}0 \\
1.000 \\
1.000 \\
1.000 \\
1.000 \\
1.000 \\
1.000 \\
1.000\end{array}$ & $\begin{array}{l}11950.953 \\
11943.651 \\
11942.282 \\
11939.297 \\
11937.953 \\
11930.595 \\
11929.944\end{array}$ & $\begin{array}{r}-0.022 \\
0.004 \\
0.002 \\
0.010 \\
0.017 \\
-0.008 \\
-0.016\end{array}$ & $\begin{array}{r}0.001 \\
-0.003 \\
0.0 \\
0.001 \\
-0.001 \\
0.0 \\
-0.002\end{array}$ \\
\hline $\begin{array}{l}40 \\
11 / 2 \\
9 / 2 \\
5 / 2 \\
7 / 2\end{array}$ & $\begin{array}{l}- \\
- \\
-\end{array}$ & $\begin{array}{l}- \\
9 / 2 \\
7 / 2 \\
3 / 2 \\
5 / 2\end{array}$ & & $\begin{array}{r}0.000 \\
1.000 \\
1.000 \\
1.000 \\
1.000\end{array}$ & $\begin{array}{l}13791.514 \\
13790.702 \\
13790.161 \\
13789.377\end{array}$ & $\begin{array}{r}0.003 \\
-0.002 \\
-0.004 \\
-0.003\end{array}$ & $\begin{array}{l}0.001 \\
0.0 \\
0.001 \\
0.0\end{array}$ \\
\hline $\begin{array}{l}411 / 2 \\
11 / 2 \\
5 / 2 \\
9 / 2 \\
7 / 2\end{array}$ & $\begin{array}{l}- \\
- \\
-\end{array}$ & $\begin{array}{l}- \\
9 / 2 \\
3 / 2 \\
7 / 2 \\
5 / 2\end{array}$ & & $\begin{array}{r}1.000 \\
1.000 \\
1.000 \\
1.000\end{array}$ & $\begin{array}{l}13316.996 \\
13315.965 \\
13315.510 \\
13314.526\end{array}$ & $\begin{array}{r}0.002 \\
-0.037 \\
-0.019 \\
-0.015\end{array}$ & $\begin{array}{l}0.001 \\
0.0 \\
-0.001 \\
0.001\end{array}$ \\
\hline $\begin{array}{c}4{ }^{1}{ }^{3} \\
5 / 2 \\
7 / 2 \\
11 / 2 \\
5 / 2 \\
9 / 2 \\
7 / 2 \\
9 / 2\end{array}$ & $\begin{array}{l}- \\
- \\
- \\
- \\
- \\
-\end{array}$ & $\begin{array}{l}- \\
5 / 2 \\
7 / 2 \\
9 / 2 \\
3 / 2 \\
7 / 2 \\
5 / 2 \\
9 / 2\end{array}$ & 3 & $\begin{array}{l}1.000 \\
1.000 \\
1.000 \\
1.000 \\
1.000 \\
1.000 \\
1.000 \\
1.000\end{array}$ & $\begin{array}{l}16234.535 \\
16229.625 \\
16228.532 \\
16228.378 \\
16226.922 \\
16226.773 \\
16220.802\end{array}$ & $\begin{array}{r}0.016 \\
-0.015 \\
0.001 \\
0.0 \\
0.002 \\
0.001 \\
0.012\end{array}$ & $\begin{array}{c}-0.006 \\
-0.005 \\
-0.001 \\
0.0 \\
-0.001 \\
0.0 \\
-0.004\end{array}$ \\
\hline $\begin{array}{l}42^{2}{ }^{3} \\
5 / 2 \\
11 / 2 \\
7 / 2 \\
9 / 2\end{array}$ & $\begin{array}{l}- \\
- \\
-\end{array}$ & $\begin{array}{l}- \\
3 / 2 \\
9 / 2 \\
5 / 2 \\
7 / 2\end{array}$ & & $\begin{array}{ll}2 & 1.000 \\
& 1.000 \\
& 1.000 \\
& 1.000\end{array}$ & $\begin{array}{l}14982.411 \\
14980.599 \\
14977.282 \\
14975.484\end{array}$ & $\begin{array}{l}0.027 \\
0.005 \\
0.014 \\
0.018\end{array}$ & $\begin{array}{l}-0.002 \\
0.0 \\
0.001 \\
-0.002\end{array}$ \\
\hline $\begin{array}{c}42^{2} 2^{2} \\
5 / 2 \\
11 / 2 \\
7 / 2 \\
7 / 2 \\
9 / 2\end{array}$ & $\begin{array}{l}- \\
- \\
-\end{array}$ & $\begin{array}{l}- \\
3 / 2 \\
9 / 2 \\
5 / 2 \\
7 / 2 \\
7 / 2\end{array}$ & & $\begin{array}{r}1.000 \\
1.000 \\
1.000 \\
1.000 \\
1.000\end{array}$ & $\begin{array}{l}16295.698 \\
16294.015 \\
16291.359 \\
16290.709 \\
16289.683\end{array}$ & $\begin{array}{r}0.015 \\
0.003 \\
0.002 \\
-0.005 \\
0.008\end{array}$ & $\begin{array}{r}0.001 \\
0.002 \\
-0.002 \\
-0.003 \\
-0.001\end{array}$ \\
\hline $\begin{array}{l}45^{3} 2^{2} \\
11 / 2 \\
7 / 2 \\
9 / 2\end{array}$ & $\begin{array}{l}- \\
- \\
-\end{array}$ & $\begin{array}{l}- \\
3 / 2 \\
9 / 2 \\
5 / 2 \\
7 / 2\end{array}$ & & 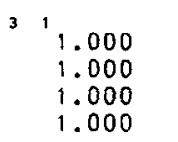 & $\begin{array}{l}15386.179 \\
15380.509 \\
15375.204 \\
15369.500\end{array}$ & $\begin{array}{r}-0.012 \\
0.011 \\
0.019 \\
-0.005\end{array}$ & $\begin{array}{r}-0.001 \\
0.003 \\
0.001 \\
-0.001\end{array}$ \\
\hline
\end{tabular}


HILLIG ET AL.

TABLE II-Continued

\begin{tabular}{|c|c|c|c|c|c|c|c|c|}
\hline \multirow{2}{*}{\multicolumn{3}{|c|}{$\begin{array}{l}\text { Transition } \\
F^{\prime}-F^{n}\end{array}$}} & \multirow{2}{*}{\multicolumn{3}{|c|}{$\begin{array}{c}\text { Normal ised } \\
\text { Weight }\end{array}$}} & \multirow{2}{*}{$\begin{array}{l}\text { Observed } \\
\text { Frequency }\end{array}$} & \multicolumn{2}{|c|}{ Residuals } \\
\hline & & & & & & & Without $x_{a b}$ & with $x_{a b}$ \\
\hline $\begin{array}{l}4{ }^{3} \\
5 / 2 \\
11 / 2 \\
7 / 2 \\
9 / 2\end{array}$ & $\begin{array}{l}- \\
- \\
-\end{array}$ & $\begin{array}{l}- \\
3 / 2 \\
9 / 2 \\
5 / 2 \\
7 / 2\end{array}$ & 3 & 3 & $\begin{array}{l}1.000 \\
0.063 \\
1.000 \\
1.000\end{array}$ & $\begin{array}{l}15516.770 \\
15511.136 \\
15505.929 \\
15500.282\end{array}$ & $\begin{array}{r}-0.011 \\
0.001 \\
0.007 \\
-0.004\end{array}$ & $\begin{array}{l}0.0 \\
-0.002 \\
-0.002 \\
0.002\end{array}$ \\
\hline $\begin{array}{l}49^{3} \\
9 / 2 \\
7 / 2 \\
11 / 2 \\
5 / 2\end{array}$ & $\begin{array}{l}- \\
- \\
-\end{array}$ & $\begin{array}{l}- \\
9 / 2 \\
7 / 2 \\
11 / 2 \\
5 / 2\end{array}$ & 4 & 1 & $\begin{array}{l}1.000 \\
1.000 \\
1.000 \\
1.000\end{array}$ & $\begin{array}{l}7457.029 \\
7454.686 \\
7450.379 \\
7448.037\end{array}$ & $\begin{array}{l}-0.008 \\
-0.019 \\
-0.005 \\
-0.017\end{array}$ & $\begin{array}{l}0.001 \\
0.001 \\
0.0 \\
0.001\end{array}$ \\
\hline $\begin{array}{l}22 / 2 \\
3 / 2 \\
5 / 2 \\
3 / 2 \\
7 / 2\end{array}$ & $\begin{array}{l}- \\
- \\
- \\
-\end{array}$ & $\begin{array}{l}- \\
5 / 2 \\
5 / 2 \\
3 / 2 \\
3 / 2 \\
7 / 2\end{array}$ & 2 & 0 & $\begin{array}{l}2 \\
1.000 \\
1.000 \\
1.000 \\
1.000 \\
1.000\end{array}$ & $\begin{array}{l}10652.225 \\
10643.245 \\
10642.867 \\
10633.887 \\
10626.691\end{array}$ & $\begin{array}{l}-0.012 \\
-0.070 \\
-0.035 \\
-0.092 \\
-0.027\end{array}$ & $\begin{array}{l}0.0 \\
-0.001 \\
0.001 \\
0.0 \\
0.001\end{array}$ \\
\hline $\begin{array}{l}32^{2} 2^{2} \\
5 / 2 \\
9 / 2 \\
3 / 2\end{array}$ & $\begin{array}{l}- \\
- \\
-\end{array}$ & $\begin{array}{l}- \\
7 / 2 \\
5 / 2 \\
9 / 2 \\
3 / 2\end{array}$ & 3 & 0 & $\begin{array}{r}1.000 \\
1.000 \\
1.000 \\
1.000\end{array}$ & $\begin{array}{l}11251.873 \\
11245.389 \\
11238.051 \\
11231.586\end{array}$ & $\begin{array}{l}-0.013 \\
-0.033 \\
-0.011 \\
-0.016\end{array}$ & $\begin{array}{l}-0.002 \\
0.0 \\
0.001 \\
0.002\end{array}$ \\
\hline $\begin{array}{l}42^{2} 3 \\
11 / 2 \\
5 / 2\end{array}$ & - & $\begin{array}{r}- \\
11 / 2 \\
5 / 2\end{array}$ & 4 & . & $\begin{array}{l}1.000 \\
0.063\end{array}$ & $\begin{array}{l}12427.138 \\
12423.834\end{array}$ & $\begin{array}{r}-0.007 \\
0.013\end{array}$ & $\begin{array}{r}0.002 \\
-0.002\end{array}$ \\
\hline$b-t y p e$ & $t$ & ansit & i & ns & & & & \\
\hline $\begin{array}{l}13^{1 / 2} \\
5 / 2 \\
1 / 2\end{array}$ & $\begin{array}{l}- \\
-\end{array}$ & $\begin{array}{l}- \\
3 / 2 \\
3 / 2 \\
3 / 2\end{array}$ & 0 & 0 & $\begin{array}{r}1.000 \\
1.000 \\
1.000\end{array}$ & $\begin{array}{l}6016.495 \\
6013.644 \\
6011.465\end{array}$ & $\begin{array}{l}0.054 \\
0.008 \\
0.081\end{array}$ & $\begin{array}{l}0.0 \\
0.0 \\
-0.001\end{array}$ \\
\hline $\begin{array}{l}21 / 2 \\
7 / 2 \\
5 / 2 \\
3 / 2 \\
5 / 2 \\
3 / 2 \\
1 / 2\end{array}$ & $\begin{array}{l}- \\
\overline{-} \\
- \\
- \\
-\end{array}$ & $\begin{array}{l}- \\
5 / 2 \\
3 / 2 \\
1 / 2 \\
5 / 2 \\
3 / 2 \\
1 / 2\end{array}$ & 1 & 0 & $\begin{array}{r}1.000 \\
1.000 \\
1.000 \\
1.000 \\
1.000 \\
1.000\end{array}$ & $\begin{array}{l}9030.653 \\
9033.506 \\
9018.011 \\
9021.011 \\
9040.422 \\
9027.659\end{array}$ & $\begin{array}{l}0.007 \\
0.046 \\
0.091 \\
0.009 \\
0.066 \\
0.074\end{array}$ & $\begin{array}{l}0.001 \\
0.0 \\
0.002 \\
-0.001 \\
-0.001 \\
0.0\end{array}$ \\
\hline $\begin{array}{l}3{ }^{1} 2^{3} \\
7 / 2 \\
5 / 2 \\
3 / 2\end{array}$ & $\begin{array}{l}- \\
- \\
-\end{array}$ & $\begin{array}{l}- \\
7 / 2 \\
5 / 2 \\
3 / 2 \\
1 / 2\end{array}$ & 2 & 0 & $\begin{array}{r}1.000 \\
1.000 \\
1.000 \\
1.000\end{array}$ & $\begin{array}{l}11737.009 \\
11737.150 \\
11733.824 \\
11733.772\end{array}$ & $\begin{array}{r}0.005 \\
0.028 \\
-0.001 \\
0.047\end{array}$ & $\begin{array}{r}0.001 \\
0.002 \\
-0.001 \\
0.001\end{array}$ \\
\hline $\begin{array}{l}41 / 2 \\
11 / 2 \\
9 / 2 \\
7 / 2 \\
5 / 2\end{array}$ & $\begin{array}{l}- \\
- \\
-\end{array}$ & $\begin{array}{l}- \\
9 / 2 \\
7 / 2 \\
5 / 2 \\
3 / 2\end{array}$ & 3 & 0 & $\begin{array}{l}1.000 \\
1.000 \\
1.000 \\
1.000\end{array}$ & $\begin{array}{l}14331.411 \\
14330.833 \\
14329.423 \\
14329.992\end{array}$ & $\begin{array}{l}0.003 \\
0.006 \\
0.0 \\
0.009\end{array}$ & $\begin{array}{l}0.001 \\
0.0 \\
0.0 \\
0.0\end{array}$ \\
\hline $\begin{array}{l}30{ }^{3} \\
9 / 2 \\
7 / 2\end{array}$ & $\begin{array}{l}- \\
-\end{array}$ & $\begin{array}{l}- \\
7 / 2 \\
5 / 2\end{array}$ & 2 & 1 & $\begin{array}{r}1.000 \\
1.000\end{array}$ & $\begin{array}{l}9083.556 \\
9079.395\end{array}$ & $\begin{array}{l}0.002 \\
0.009\end{array}$ & $\begin{array}{l}0.002 \\
0.0\end{array}$ \\
\hline
\end{tabular}


TABLE II-Continued

\begin{tabular}{|c|c|c|c|c|c|c|c|}
\hline \multirow{2}{*}{\multicolumn{3}{|c|}{$\begin{array}{l}\text { Transition } \\
F^{\prime} F^{n}\end{array}$}} & \multirow{2}{*}{\multicolumn{2}{|c|}{$\begin{array}{c}\text { Normalised } \\
\text { Weight }\end{array}$}} & \multirow{2}{*}{$\begin{array}{l}\text { Observed } \\
\text { Frequency }\end{array}$} & \multicolumn{2}{|c|}{ Residuals } \\
\hline & & & & & & without $x_{a b}$ & With $x_{a b}$ \\
\hline $\begin{array}{l}5 / 2 \\
3 / 2\end{array}$ & - & $\begin{array}{l}3 / 2 \\
1 / 2\end{array}$ & & $\begin{array}{l}1.000 \\
1.000\end{array}$ & $\begin{array}{l}9078.939 \\
9083.140\end{array}$ & $\begin{array}{r}-0.005 \\
0.021\end{array}$ & $\begin{array}{l}0.0 \\
0.001\end{array}$ \\
\hline $\begin{array}{l}4 \frac{0}{4} \\
11 / 2 \\
9 / 2 \\
7 / 2 \\
5 / 2\end{array}$ & $\begin{array}{l}- \\
- \\
-\end{array}$ & $\begin{array}{l}- \\
9 / 2 \\
7 / 2 \\
5 / 2 \\
3 / 2\end{array}$ & 3 & $\begin{array}{r}1.000 \\
1.000 \\
1.000 \\
1.000\end{array}$ & $\begin{array}{l}12777.100 \\
12775.382 \\
12774.483 \\
12776.134\end{array}$ & $\begin{array}{r}0.003 \\
-0.024 \\
-0.016 \\
-0.050\end{array}$ & $\begin{array}{l}0.003 \\
0.002 \\
0.004 \\
0.001\end{array}$ \\
\hline $\begin{array}{l}2{ }^{2} / 2 \\
5 / 2 \\
5 / 2 \\
3 / 2 \\
3 / 2 \\
7 / 2 \\
1 / 2\end{array}$ & $\begin{array}{l}- \\
- \\
- \\
- \\
-\end{array}$ & $\begin{array}{l}- \\
5 / 2 \\
3 / 2 \\
1 / 2 \\
3 / 2 \\
5 / 2 \\
1 / 2\end{array}$ & 1 & $\begin{array}{r}1.000 \\
1.000 \\
0.063 \\
0.063 \\
1.000 \\
1.000\end{array}$ & $\begin{array}{l}15960.102 \\
15957.248 \\
15952.930 \\
15947.900 \\
15947.030 \\
15939.931\end{array}$ & $\begin{array}{r}0.012 \\
-0.037 \\
-0.084 \\
-0.057 \\
-0.006 \\
-0.045\end{array}$ & $\begin{array}{l}0.0 \\
-0.003 \\
0.0 \\
-0.001 \\
-0.002 \\
0.0\end{array}$ \\
\hline $\begin{array}{l}25^{2}{ }^{1} \\
3 / 2 \\
5 / 2 \\
7 / 2 \\
1 / 2 \\
3 / 2\end{array}$ & $\begin{array}{l}- \\
- \\
- \\
-\end{array}$ & $\begin{array}{l}- \\
5 / 2 \\
1 / 2 \\
3 / 2 \\
5 / 2 \\
1 / 2 \\
3 / 2\end{array}$ & 1 & $\begin{array}{r}1.000 \\
1.000 \\
1.000 \\
1.000 \\
1.000 \\
1.000\end{array}$ & $\begin{array}{l}15037.231 \\
15035.997 \\
15027.593 \\
15024.731 \\
15023.658 \\
15018.609\end{array}$ & $\begin{array}{r}0.014 \\
-0.021 \\
0.029 \\
-0.013 \\
0.067 \\
-0.033\end{array}$ & $\begin{array}{r}-0.001 \\
0.002 \\
0.002 \\
0.002 \\
0.0 \\
-0.003\end{array}$ \\
\hline $7 / 2^{2}$ & - & - & 2 & $2 \quad 1.000$ & 5630.540 & 0.010 & 0.0 \\
\hline $\begin{array}{c}45^{1 / 2}{ }^{3} \\
11 / 2 \\
7 / 2 \\
9 / 2\end{array}$ & $\begin{array}{l}- \\
- \\
-\end{array}$ & $\begin{array}{l}- \\
3 / 2 \\
9 / 2 \\
5 / 2 \\
7 / 2\end{array}$ & 3 & $\begin{array}{ll}22 & 1.000 \\
& 1.000 \\
1.000 & 1.000\end{array}$ & $\begin{array}{l}10546.446 \\
10543.739 \\
10538.719 \\
10535.986\end{array}$ & $\begin{array}{l}0.011 \\
0.008 \\
0.013 \\
0.009\end{array}$ & $\begin{array}{l}0.001 \\
0.0 \\
0.0 \\
-0.001\end{array}$ \\
\hline $\begin{array}{l}73^{4} \\
17 / 2 \\
15 / 2 \\
13 / 2 \\
11 / 2\end{array}$ & $\begin{array}{l}- \\
- \\
-\end{array}$ & $\begin{array}{l}- \\
15 / 2 \\
13 / 2 \\
11 / 2 \\
9 / 2\end{array}$ & 6 & $\begin{array}{r}3 . \\
1.000 \\
1.000 \\
1.000 \\
1.000\end{array}$ & $\begin{array}{l}11978.406 \\
11972.217 \\
11973.061 \\
11980.480\end{array}$ & $\begin{array}{l}0.002 \\
0.680 \\
0.045 \\
0.607\end{array}$ & $\begin{array}{l}-0.001 \\
-0.001 \\
0.0 \\
0.001\end{array}$ \\
\hline $\begin{array}{l}73^{3} 5 \\
17 / 2 \\
15 / 2 \\
13 / 2 \\
11 / 2\end{array}$ & $\begin{array}{l}- \\
- \\
-\end{array}$ & $\begin{array}{l}- \\
15 / 2 \\
13 / 2 \\
11 / 2 \\
9 / 2\end{array}$ & 6 & $\begin{array}{r}42 \\
1.000 \\
1.000 \\
1.000 \\
1.000\end{array}$ & $\begin{array}{l}8513.626 \\
8505.080 \\
8507.052 \\
8514.996\end{array}$ & $\begin{array}{l}-0.001 \\
-0.228 \\
-0.031 \\
-0.399\end{array}$ & $\begin{array}{l}0.001 \\
0.0 \\
0.001 \\
0.0\end{array}$ \\
\hline $\begin{array}{l}3 / 2 \\
7 / 2 \\
5 / 2 \\
9 / 2 \\
3 / 2\end{array}$ & $\begin{array}{l}- \\
- \\
-\end{array}$ & $\begin{array}{l}7 / 2 \\
5 / 2 \\
9 / 2 \\
3 / 2\end{array}$ & 3 & $\begin{array}{r}1.000 \\
1.000 \\
1.000 \\
1.000 \\
1.000\end{array}$ & $\begin{array}{l}5560.939 \\
5557.337 \\
5553.257 \\
5549.651\end{array}$ & $\begin{array}{l}-0.004 \\
-0.019 \\
-0.005 \\
-0.008\end{array}$ & $\begin{array}{l}0.0 \\
0.002 \\
0.001 \\
0.0\end{array}$ \\
\hline $\begin{array}{c}41 / 23 \\
11 / 2 \\
5 / 2 \\
7 / 2 \\
9 / 2 \\
7 / 2 \\
9 / 2 \\
11 / 2\end{array}$ & $\begin{array}{l}- \\
- \\
- \\
- \\
- \\
-\end{array}$ & $\begin{array}{l}- \\
9 / 2 \\
7 / 2 \\
9 / 2 \\
9 / 2 \\
7 / 2 \\
7 / 2 \\
11 / 2\end{array}$ & 4 & $\begin{array}{r}1.000 \\
1.000 \\
1.000 \\
1.000 \\
1.000 \\
1.000 \\
1.000\end{array}$ & $\begin{array}{l}8004.890 \\
8002.497 \\
7999.865 \\
7997.160 \\
7994.732 \\
7992.026 \\
7990.276\end{array}$ & $\begin{array}{r}-0.011 \\
0.002 \\
-0.015 \\
0.0 \\
-0.016 \\
-0.002 \\
-0.005\end{array}$ & $\begin{array}{l}0.003 \\
-0.002 \\
-0.002 \\
0.0 \\
0.0 \\
0.002 \\
0.0\end{array}$ \\
\hline
\end{tabular}


TABLE II-Continued

\begin{tabular}{|c|c|c|c|c|c|c|c|c|}
\hline \multirow{2}{*}{\multicolumn{3}{|c|}{$\begin{array}{l}\text { Transition } \\
F^{\prime}-F^{n}\end{array}$}} & \multirow{2}{*}{\multicolumn{3}{|c|}{$\begin{array}{l}\text { Normalised } \\
\text { Weight }\end{array}$}} & \multirow{2}{*}{$\begin{array}{l}\text { Observed } \\
\text { Frequency }\end{array}$} & \multicolumn{2}{|c|}{ Residuals } \\
\hline & & & & & & & without $x_{a b}$ & With $x_{a b}$ \\
\hline $\begin{array}{l}5 / 2 \\
9 / 2 \\
7 / 2\end{array}$ & - & $\begin{array}{r}5 / 2 \\
11 / 2 \\
5 / 2\end{array}$ & & & $\begin{array}{l}1.000 \\
1.000 \\
1.000\end{array}$ & $\begin{array}{l}7987.869 \\
7982.545 \\
7980.103\end{array}$ & $\begin{array}{r}-0.003 \\
0.005 \\
-0.021\end{array}$ & $\begin{array}{r}0.001 \\
-0.004 \\
0.003\end{array}$ \\
\hline $\begin{array}{l}25^{2}{ }^{\circ} \\
5 / 2 \\
5 / 2 \\
3 / 2 \\
3 / 2 \\
7 / 2 \\
3 / 2 \\
7 / 2 \\
1 / 2 \\
1 / 2\end{array}$ & $\begin{array}{l}- \\
- \\
- \\
- \\
- \\
-\end{array}$ & $\begin{array}{l}- \\
5 / 2 \\
3 / 2 \\
7 / 2 \\
5 / 2 \\
3 / 2 \\
5 / 2 \\
1 / 2 \\
7 / 2 \\
3 / 2 \\
1 / 2\end{array}$ & 2 & 1 & $\begin{array}{l}1.000 \\
1.000 \\
1.000 \\
1.000 \\
1.000 \\
1.000 \\
1.000 \\
1.000 \\
1.000 \\
1.000\end{array}$ & $\begin{array}{l}6886.747 \\
6884.739 \\
6883.948 \\
6877.396 \\
6875.388 \\
6873.680 \\
6872.571 \\
6870.881 \\
6862.388 \\
6859.571\end{array}$ & $\begin{array}{l}0.021 \\
0.024 \\
0.018 \\
-0.003 \\
0.001 \\
0.008 \\
0.0 \\
0.005 \\
0.039 \\
0.039\end{array}$ & $\begin{array}{l}0.0 \\
0.001 \\
-0.002 \\
-0.002 \\
0.0 \\
0.002 \\
0.001 \\
0.0 \\
-0.001 \\
0.0\end{array}$ \\
\hline $\begin{array}{l}2{ }^{2} / 2 \\
5 / 2 \\
3 / 2 \\
1 / 2\end{array}$ & & $\begin{array}{l}- \\
7 / 2 \\
5 / 2 \\
3 / 2 \\
1 / 2\end{array}$ & 2 & 1 & $\begin{array}{r}1.000 \\
1.000 \\
1.000 \\
1.000\end{array}$ & $\begin{array}{l}8987.646 \\
9009.790 \\
8993.895 \\
8971.917\end{array}$ & $\begin{array}{r}-0.036 \\
-0.009 \\
-0.084 \\
0.029\end{array}$ & $\begin{array}{r}-0.005 \\
-0.004 \\
-0.004 \\
0.002\end{array}$ \\
\hline $\begin{array}{l}32^{2} 2^{2} \\
5 / 2 \\
9 / 2 \\
3 / 2\end{array}$ & & $\begin{array}{l}- \\
7 / 2 \\
5 / 2 \\
9 / 2 \\
3 / 2\end{array}$ & 3 & 1 & $\begin{array}{r}1.000 \\
1.000 \\
1.000 \\
1.000\end{array}$ & $\begin{array}{l}10236.554 \\
10230.491 \\
10223.634 \\
10217.556\end{array}$ & $\begin{array}{l}-0.034 \\
-0.050 \\
-0.013 \\
-0.065\end{array}$ & $\begin{array}{l}0.002 \\
0.0 \\
0.0 \\
0.0\end{array}$ \\
\hline $\begin{array}{l}37^{2} / 2 \\
5 / 2 \\
9 / 2 \\
3 / 2\end{array}$ & & $\begin{array}{l}- \\
7 / 2 \\
5 / 2 \\
9 / 2 \\
3 / 2\end{array}$ & & 1 & $\begin{array}{r}1.000 \\
1.000 \\
1.000 \\
1.000\end{array}$ & $\begin{array}{l}6463.702 \\
6460.196 \\
6456.203 \\
6452.690\end{array}$ & $\begin{array}{l}-0.005 \\
0.0 \\
0.003 \\
-0.014\end{array}$ & $\begin{array}{l}0.001 \\
0.0 \\
0.001 \\
0.0\end{array}$ \\
\hline $\begin{array}{l}42^{2} 2^{2} \\
5 / 2 \\
7 / 2 \\
9 / 2 \\
7 / 2 \\
9 / 2 \\
11 / 2 \\
5 / 2 \\
9 / 2 \\
7 / 2\end{array}$ & & $\begin{array}{r}- \\
9 / 2 \\
7 / 2 \\
9 / 2 \\
9 / 2 \\
7 / 2 \\
7 / 2 \\
11 / 2 \\
5 / 2 \\
11 / 2 \\
5 / 2\end{array}$ & & 1 & $\begin{array}{l}1.000 \\
1.000 \\
1.000 \\
1.000 \\
1.000 \\
1.000 \\
1.000 \\
1.000 \\
1.000 \\
1.000\end{array}$ & $\begin{array}{l}6529.412 \\
6527.775 \\
6527.489 \\
6526.463 \\
6524.783 \\
6523.758 \\
6521.683 \\
6520.010 \\
6518.733 \\
6517.018\end{array}$ & $\begin{array}{r}-0.010 \\
0.019 \\
-0.012 \\
0.001 \\
0.001 \\
0.016 \\
0.002 \\
0.001 \\
0.013 \\
-0.016\end{array}$ & $\begin{array}{l}0.003 \\
-0.002 \\
-0.002 \\
0.0 \\
0.0 \\
0.003 \\
0.001 \\
0.0 \\
-0.003 \\
0.002\end{array}$ \\
\hline $\begin{array}{l}42^{3} \\
5 / 2 \\
7 / 2 \\
9 / 2 \\
7 / 2 \\
9 / 2 \\
11 / 2 \\
5 / 2\end{array}$ & & $\begin{array}{l}- \\
7 / 2 \\
9 / 2 \\
9 / 2 \\
7 / 2 \\
7 / 2 \\
11 / 2 \\
5 / 2\end{array}$ & & 1 & $\begin{array}{l}1.000 \\
1.000 \\
1.000 \\
1.000 \\
1.000 \\
1.000 \\
1.000\end{array}$ & $\begin{array}{l}11898.422 \\
11898.297 \\
11896.527 \\
11893.247 \\
11891.480 \\
11887.239 \\
11884.004\end{array}$ & $\begin{array}{r}0.020 \\
-0.022 \\
0.001 \\
-0.020 \\
0.005 \\
-0.009 \\
0.001\end{array}$ & $\begin{array}{l}0.001 \\
-0.001 \\
-0.001 \\
-0.001 \\
0.003 \\
0.0 \\
0.0\end{array}$ \\
\hline $\begin{array}{l}6 / 2 \\
15 / 2 \\
13 / 2 \\
11 / 2 \\
9 / 2\end{array}$ & & $\begin{array}{l}- \\
15 / 2 \\
13 / 2 \\
11 / 2 \\
9 / 2\end{array}$ & & 1 & $\begin{array}{l}1.000 \\
1.000 \\
1.000 \\
1.000\end{array}$ & $\begin{array}{l}9042.412 \\
9046.084 \\
9045.214 \\
9041.543\end{array}$ & $\begin{array}{l}0.002 \\
0.002 \\
0.0 \\
0.001\end{array}$ & $\begin{array}{l}0.001 \\
0.002 \\
0.0 \\
0.002\end{array}$ \\
\hline
\end{tabular}


TABLE II-Continued

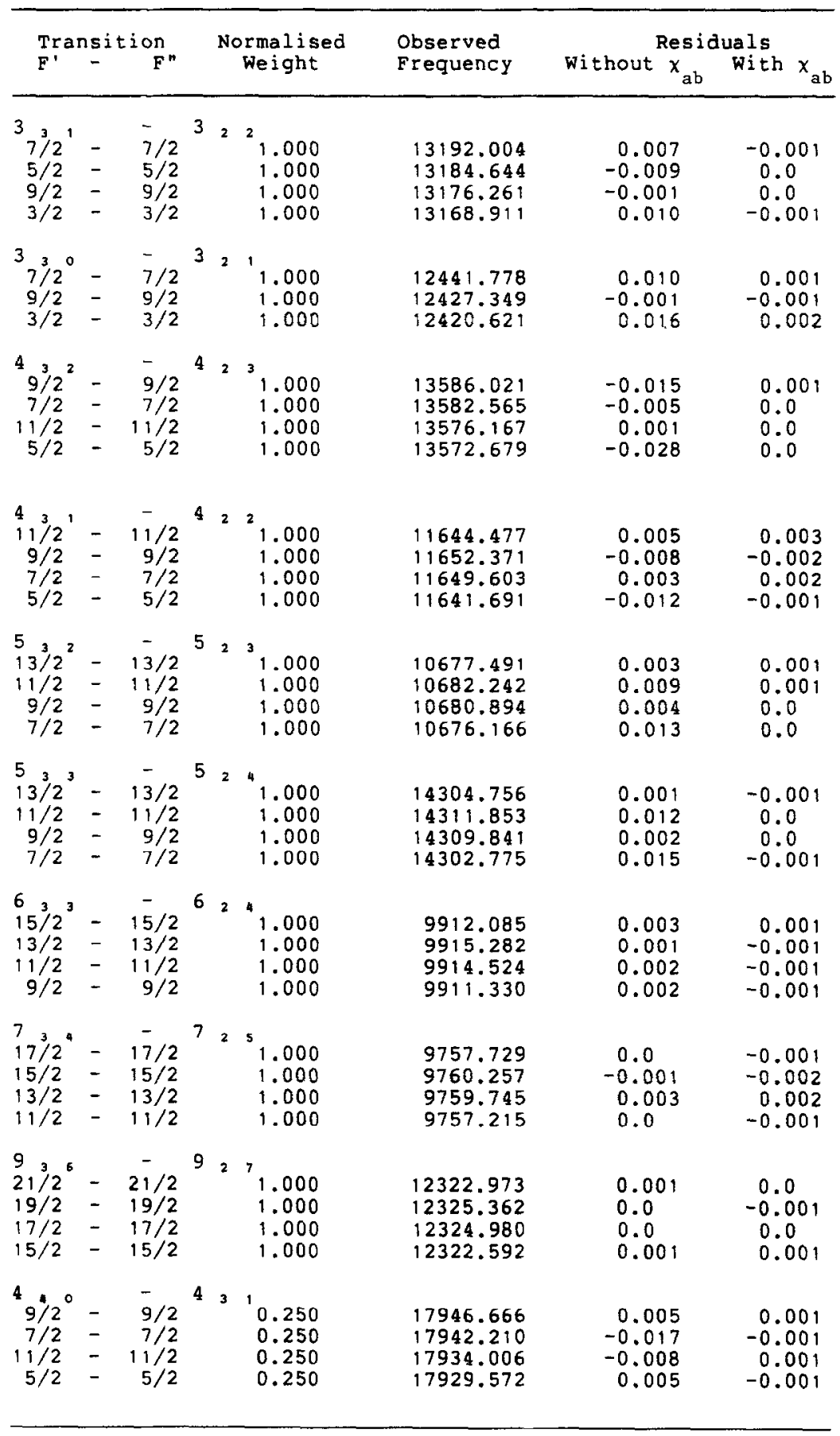


TABLE II-Continued

\begin{tabular}{|c|c|c|c|c|c|c|c|}
\hline \multicolumn{3}{|c|}{ Transition } & \multirow{2}{*}{\multicolumn{2}{|c|}{$\begin{array}{c}\text { Normal ised } \\
\text { Weight }\end{array}$}} & \multirow{2}{*}{$\begin{array}{l}\text { Observed } \\
\text { Frequency }\end{array}$} & \multicolumn{2}{|c|}{ Residuals } \\
\hline$F^{\prime}$ & & $F^{n}$ & & & & Without $x_{a b}$ & with $x_{a b}$ \\
\hline $\begin{array}{r}49 / 2 \\
9 / 2 \\
7 / 2 \\
11 / 2 \\
5 / 2\end{array}$ & - & $\begin{array}{r}- \\
9 / 2 \\
7 / 2 \\
11 / 2 \\
5 / 2\end{array}$ & 4 & $\begin{array}{ll}32 & \\
& 0.250 \\
& 0.250 \\
& 0.250 \\
& 0.250\end{array}$ & $\begin{array}{l}18097.417 \\
18092.897 \\
18084.570 \\
18080.070\end{array}$ & $\begin{array}{r}0.004 \\
-0.017 \\
-0.007 \\
0.007\end{array}$ & $\begin{array}{l}0.0 \\
0.003 \\
0.004 \\
0.001\end{array}$ \\
\hline $\begin{array}{l}6 / 42^{2} \\
15 / 2 \\
13 / 2 \\
11 / 2 \\
9 / 2\end{array}$ & 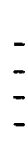 & $\begin{array}{l}-\overline{15 / 2} \\
13 / 2 \\
11 / 2 \\
9 / 2\end{array}$ & 6 & 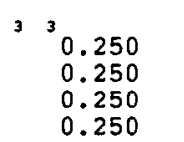 & $\begin{array}{l}16735.773 \\
16741.390 \\
16739.916 \\
16734.900\end{array}$ & $\begin{array}{r}-0.001 \\
0.225 \\
0.029 \\
0.399\end{array}$ & $\begin{array}{c}-0.001 \\
-0.003 \\
-0.002 \\
0.0\end{array}$ \\
\hline $\begin{array}{l}6 / 5 / 2^{3} \\
13 / 2 \\
11 / 2 \\
9 / 2\end{array}$ & - & $\begin{array}{l}- \\
15 / 2 \\
13 / 2 \\
11 / 2 \\
9 / 2\end{array}$ & 6 & 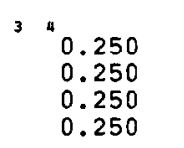 & $\begin{array}{l}18193.903 \\
18199.427 \\
18198.600 \\
18191.818\end{array}$ & $\begin{array}{r}0.003 \\
-0.685 \\
-0.041 \\
-0.616\end{array}$ & $\begin{array}{l}0.002 \\
0.0 \\
0.002 \\
-0.003\end{array}$ \\
\hline $\begin{array}{l}9 / 4 / 5 \\
21 / 2 \\
19 / 2 \\
17 / 2 \\
15 / 2\end{array}$ & & $\begin{array}{l}- \\
21 / 2 \\
19 / 2 \\
17 / 2 \\
15 / 2\end{array}$ & $y$ & 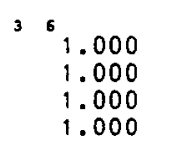 & $\begin{array}{l}13158.899 \\
13160.825 \\
13160.519 \\
13158.590\end{array}$ & $\begin{array}{r}0.001 \\
0.001 \\
0.003 \\
-0.001\end{array}$ & $\begin{array}{l}0.0 \\
0.0 \\
0.002 \\
-0.001\end{array}$ \\
\hline $\begin{array}{l}10 \\
23 / 2 \\
21 / 2 \\
19 / 2 \\
17 / 2\end{array}$ & - & $\begin{array}{l}- \\
23 / 2 \\
21 / 2 \\
19 / 2 \\
17 / 2\end{array}$ & & $\begin{array}{r}3 \quad 1.000 \\
1.000 \\
1.000 \\
1.000\end{array}$ & $\begin{array}{l}12833.416 \\
12835.093 \\
12834.851 \\
12833.171\end{array}$ & $\begin{array}{r}0.002 \\
0.001 \\
0.001 \\
-0.002\end{array}$ & $\begin{array}{l}0.001 \\
0.0 \\
0.0 \\
-0.002\end{array}$ \\
\hline $\begin{array}{l}11 \\
25 / 2 \\
23 / 2 \\
21 / 2 \\
19 / 2\end{array}$ & & $\begin{array}{l}- \\
25 / 2 \\
23 / 2 \\
21 / 2 \\
19 / 2\end{array}$ & & $\begin{array}{ll}3 \quad 8 & 1.000 \\
& 1.000 \\
& 1.000 \\
& 1.000\end{array}$ & $\begin{array}{l}13558.512 \\
13560.172 \\
13559.952 \\
13558.296\end{array}$ & $\begin{array}{l}0.0 \\
0.002 \\
-0.001 \\
0.003\end{array}$ & $\begin{array}{r}-0.001 \\
0.001 \\
-0.002 \\
0.002\end{array}$ \\
\hline
\end{tabular}

MHz), they are nevertheless significant. However, for four transitions, namely $7_{34} \leftarrow$ $6_{43}, 7_{35} \leftarrow 6_{42}, 6_{43} \leftarrow 6_{34}$, and $6_{42} \leftarrow 6_{33}$, they are large enough to be detectable even with a Stark-modulated spectrometer. This is the result of the two near degeneracies $5_{51}-6_{43}$ and $6_{42}-5_{50}$, both of which are less than $100 \mathrm{MHz}$. Since these transitions can be expected to have a large effect on the precision of $x_{a b}$, and since one of the objectives was to see whether such transitions are necessary to obtain an accurate $x_{a b}$, another fit was done omitting them. The results are also in Table I; it is clear that an excellent $x_{a b}$ was obtained, albeit at reduced precision. Thus, this coupling constant can be determined from frequency measurements of sufficiently high precision in the absence of perturbing near degeneracies. However, the effects of centrifugal distortion may be comparable to the contributions from $x_{a b}$ and must be properly taken into account. Of course, a large value of $x_{a b}$ is desirable, necessitating propitious values for $\theta_{z a}$ and $x_{a a}-x_{b b}$ since $2 x_{a b}=\left(x_{a a}-x_{b b}\right) \tan 2 \theta_{z a}$.

\section{DISCUSSION}

Diagonalization of the quadrupole tensor gives the following principal values: $x_{z z}$ $=-77.46 \pm 0.1 \mathrm{MHz}, x_{x x}=38.85 \pm 0.1 \mathrm{MHz}$, and $x_{y y}=x_{c c}=38.614 \pm 0.003$ 
$\mathrm{MHz}$ (the uncertainties are estimated outside error limits). The differences between our results and those of Stone and Flygare (1) are not due to improperly determined constants in either study. Table I gives a comparison, and it is clear that there is excellent agreement. The cause must thus lie in an incorrect assumed structure in Ref. ( 1 ) and a consequent incorrect angle $\theta_{z a}$ between the $z$-principal axis and the $a$ inertial axis. We have found $\theta_{z a}$ to be $29.16 \pm 0.03^{\circ}$; in Ref. ( 1 ) it is $32.71^{\circ}$, a difference of $3.55^{\circ}$. A discrepancy this large when $\theta_{z a}$ is $\sim 25-45^{\circ}$ should have a drastic effect on $x_{a b}$. For $\theta_{z a}=32.71^{\circ},\left|x_{a b}\right|=67.2 \mathrm{MHz}$, considerably different from the experimental value of $49.489 \mathrm{MHz}$.

A simple Townes-Dailey calculation ( 8 ) gives the ionic and $\pi$-character of the C$\mathrm{Cl}$ bond assuming this is the $z$-axis of the quadrupole tensor. Since $x_{x x} \simeq x_{y y}$, the $\pi$ character is effectively nil. This is smaller than in other vinyl chlorides where the chlorine double bonded structure $\left({ }^{+} \mathrm{Cl}=\right.$ ) contributes from $2.4-5.3 \%(1)$. The ionic contribution, obtained assuming $15 \% s$-character and no $d$-character of the $\sigma$-bonding orbital of $\mathrm{Cl}$, is $\sim 17 \%$, so that the covalent character is $\sim 83 \%$. These values are much more in line with those of other vinyl chlorides (1).

\section{ACKNOWLEDGMENTS}

This work was supported by the Natural Sciences and Engineering Research Council of Canada and Grant CHE-8614340 from the National Science Foundation, Washington, DC. Use of the CRAY X-MP computer of the Atmospheric Environment Services of Canada is gratefully acknowledged. ERB was supported by the UM Summer Undergraduate Research Program (1987). We are grateful to Dr. Rick Suenram who pointed out the conflicting assignments for $\mathrm{CF}_{2}=\mathrm{CFCl}$ and Mr. P. Duffy for sume computing assistance.

RECEIVED: July 7, 1988

\section{REFERENCES}

1. R. G. Stone AND W. H. Flygare, J. Mol. Spectrosc. 32, 233-241 (1969).

2. Krishnaji And S. Chandra, Indian J. Pure Appl. Phys. 8, 634 (1970).

3. K. W. Hillig II, J. Matos, A. Scioly, AND R. L. KuCZKowsKI, Chem. Phys. Lett. 133, 359 (1987).

4. M. C. L. Gerry, W. Lewis-Bevan, And N. P. C. Westwood, J. Chem Phys. 79, 4655-4663 (1983).

5. T. J. Balle AND W. H. FlyGare, Rev. Sci. Instr. 52, 33 (1981).

6. H. M. Jemson, W. Lewis-Bevan, N. P. C. Westwood, and M. C. L. Gerry, J. Mol. Spectrosc. 118, 481-499 (1986).

7. J. K. G. WATSON, in "Vibrational Spectra and Structure, a Series of Advances" (J. R. Durig, Ed.), Vol. 6, pp. 1-89, Elsevier, New York, 1977.

8. W. GORDY AND R. L. COOK, "Microwave Molecular Spectra," 3rd ed., Wiley, New York, 1984. 